\title{
LISTA COMENTADA DE LOS PTERIDOFITOS DE ANDALUCIA
}

\author{
A. E. SALVO \& B. CABEZUDO
}

RESUMEN: La flora pteridofitica de Andalucía consta de 30 géneros, 68 especies, 9 subespecies, 7 variedades, 2 formas y 7 híbridos. Para cada especie se indica la ecologia, fitosociología, pisos bioclimáticos y corología regional.

SUMMARY: The pteridological flora from Andalucía comprehends 30 genera, 68 species, 9 subspecies, 7 varieties, 2 forms and 7 hybrids. Ecology, phytosociology, vegetation stage and regional chorology is given for each specie.

\section{INTRODUCCION}

El presente catálogo de los pteridófitos de Andalucía pretende ser una actualización del presentado en la obra de Salvo (1982). Tal como afirmaba entonces el autor era previsible el aumento del número de táxones y la ampliación de citas corológicas, a tenor de las investigaciones pteridológicas en curso de realización. Es por ello que creemos oportuno en este momento ofrecer cual es el estado actual del conocimiento de la flora pteridofítica andaluza.

Además del catálogo florístico, que queda comprendido por 30 géneros, 68 especies, 9 subespecies, 7 variedades, 2 formas y 7 híbridos, hemos acompañado a cada especie de unos escuetos comentarios ecológicos, fitosociológicos y corológicos, a los que aludimos a continuación. Para la estructuración de dichos comentarios hemos adaptado la utilizada por Rivas-Martínez, Costa, Izco \& Sáenz (1981) de la siguiente forma: 1. Autoecología. 2. Fitosociología. 3. Pisos de vegetación, indicando en cada uno la frecuencia de aparición según los indices de Willmanns \& Rasbach (1973): (VR) muy rara, (R) rara, (O) ocasional, (C) común, (VC) muy común. 4. Distribución -en Andalucía (unidades corológicas según la división corológica propuesta por Cabezudo \& al. in Salvo (1982). 5. Distribución por provincias políticas (Al: Almería; Ca: Cádiz; Co: Córdoba; Gr: Granada; Hu: Huelva; J: Jaén; Ma: Málaga; Se: Sevilla). 


\section{CATALOGO}

\section{SElAginella P.B., Prodr. Fam. Aethéogr.: 101 (1905)}

Selaginella denticulata (L.) Link, Fil. Sp.: 159 (1841)

E Lycopodium denticulatum L., Sp. Pl. 2: 1106 (1753)

- Comunidades briopteridofíticas algo esciófilas y de ambiente mesofítico proporcionado por los taludes de los bosques templados - Característica de Anogrammo-Selaginelletum (Anogrammo-Polypodietea) - Termomediterráneo (O), Mesomediterráneo (R) - Araceno-Pacense, Andevalense, Marianense, Onubense, Gaditano, Malacitano costero, Aljíbico, Hispalense, Rondense, Bermejense, Mijense, MalacitanoAxarquiense, Almijaro-Cazulense, Alpujarro-Gadorense, Almeriense Al, Ca, Co, Gr, Hu, J, Ma, Se.

ISOETES L., Sp. Pl. 2: 1100 (1753)

Isoetes durieui Bory in Compt. Rend. Acad. Scien. (Paris) 18: 1166 (1844)

- Comunidades de terófitos encharcadas - Característica de Isoetion, también en Cicendion (Isoeto-Nanojuncetea) - Termomediterráneo (R), Mesomediterráneo (O) - Araceno-Pacense, Aljíbico - Ca, $\mathrm{Hu}$.

Isoetes histrix Bory in Compt. Rend. Acad. Scien. (Paris) 18: 1167 (1844)

= Isoetes chaetureti Mendes in Agron. Lusit. 23: 7-8 (1961)

- Comunidades de terófitos períodicamente encharcadas - LotoChaetopogonetum fasciculati, característica de Radiolo-Isoetetum histricis (caracteristica de Isoetalia y de Isoeto-Nanojuncetea) - Termomediterráneo (O), Mesomediterráneo ( $R$ ) - Araceno-Pacense, Andevalense, Marianense, Onubense, Gaditano, Aljíbico, Hispalense - Ca, $\mathrm{Co}, \mathrm{Hu}$, Se.

Isoetes setacea Lam., Encycl. Méth. Bot. 3: 314 (1789)

= Isoetes delilei Rothmaler in Feddes Repert. 54: 72 (1944)

- Comunidades anfibias de charcas y lagunazos que permanecen secas durante el período estival, sobre suelos arenosos y ácidos Hyperico-Cicendietum filiformis (característica de Cicendion e Isoetalia, Isoeto-Nanojuncetea) - Termomediterráneo (R), Mesomediterráneo (O) - Marianense, Onubense - Co, Hu.

Isoetes velata A. Br. in Durieu, Expl. Sci. d'Alger Bot. 1: 19 (1850) - Comunidades pioneras de hondonadas donde permanece el agua durante la mayor parte del año - Característica de Junco-Isoetetum velatae (Cicendion, Isoeto-Nanojuncetea) - Termomediterráneo (O), Mesomediterráneo (R), Supramediterráneo (VR) - Marianense, Onubense, Aljíbico, Nevadense - Ca, Gr, Hu, Se.

Observaciones: Siguiendo a Prada (1983) se reconocen dos formas distintas de esta especie en Andalucía, la forma velata (con micrófilas de 20-40 por rizoma, de 15-30 cm de longitud) y la forma baetica (Willk.) Prada in Acta Bot. Malacitana 8: 88 (1983) (= 1. baetica Willk.) (con micrófilas de 5-12 por rizoma, de 4-10 cm de longitud).

$$
\text { EQUISETUM L., Sp. Pl.: } 1061 \text { (1753) }
$$

Equisetum telmateia Ehrh. in Hannover Mag. 31: 287 (1783)

- Comunidades higrófilas y preferentemente umbrófilas - Osmun- 
do-Alnion, Populion, Holoschoenion - Termomediterráneo (C), Mesomediterráneo (O), Supramediterráneo (O) - Araceno-Pacense, Andevalense, Marianense, Onubense, Gaditano, Aljíbico, Rondense, Mijense, Malacitano-Axarquiense, Almijaro-Cazulense, Seguro-Cazorlense, Granatense, Nevadense, Almeriense - Al, Ca, Co, Gr, Hu, J, Ma, Se.

Equisetum arvense L., Sp. P1.: 1061 (1753)

- Márgenes de remansos fluviales - Holoschoenion - Mesomediterráneo (0), Supramediterráneo (O) - Rondense, Nevadense, SeguroCazorlense - Co, Gr, J.

Equisetum ramosissimum Desf., Fl. Atl. 2: 398 (1799)

- Comunidades riparias (tarajares, adelfares, saucedas, sotos, choperas, alisedas, fresnedas, olmedas, etc), de zonas húmedas (juncales, cañaverales, etc), nitrófilas (ruderales, arvenses, etc) o incluso en comunidades psammófilas - Termomediterráneo (C), Mesomediterráneo (O), Supramediterráneo (O) - En todas las unidades corológicas - Al, Ca, Co, Gr, Hu, J, Ma, Se.

Observaciones: Molesworth (1968) cita Equisetum $x$ moorei Newman (E. hyemale $x$ E. ramosissimum) en Algeciras. En nuestra opinión este material se trata de una forma ecotípica de E. ramosissimum, de fenótipo similar al hibrido en cuestión, determinada por las especiales condiciones ecológicas de los ecosistemas arenosos costeros.

PSILOTUM Swartz in J. Bot. Schrader 1800 (2): 8 (1801)

Psilotum nudum (L.) P.B., Prodr. Aethéogr.: 112 (1805)

$\equiv$ Lycopodium nudum L., Sp. P1. 1,2: 1100 (1753)

- Fisuras de rocas - Asplenietea rupestris - Termomediterráneo (VR) - Aljíbico - Ca.

Observaciones: En Salvo (1982) se propone el rango varietal para las poblaciones europeas de esta especie, P. nudum var. molesworthae Iranzo, Prada \& Salvo, atendiendo para ello al menor tamaño y mayor grosor de los tallos aéreos.

BOTRYCHIUM Swartz in J. Bot. Schrader 1800 (2): 8 (1801)

Botrychium lunaria (L.) Swartz in J. Bot. Schrader 1800 (2): 110 (1801)

ミOsmunda lunaria L., Sp. Pl. 2: 1064 (1753)

- Pastizales higroturbosos (borreguiles) - Nardetea - Supramediterráneo (R), Oromediterráneo (R) - Nevadense, Almijaro-Cazulense - Gr, Ma.

$$
\text { OPHIOGLOSSUM L., Sp. Pl.: } 1062 \text { (1753) }
$$

Ophioglossum vulgatum L., Sp. Pl. 2: 1062 (1753)

- Praderas higrófilas y juncales oligótrofos - Característica de Molinetalia (Molinio-Arrhenatheretea), Caricetum pseudocypero-lusitanicae (Phragmitetalia, Phragmitetea) - Termomediterráneo (VR), Mesomediterráneo (R), Supramediterráneo (R) - Onubense, Nevadense, Seguro-Cazorlense - Gr, Hu, J.

Ophioglossum lusitanicum L., Sp. P1. 2: 1063 (1753)

- Comunidades de pastizales terofíticos fugaces (majadales)

- Tuberarietea, Isoeteo-Nanojuncetea - Termomediterráneo (R), Miesomediterráneo (R) - Araceno-Pacense, Andevalense, Onubense, Gaditano, Marianense, Aljíbico, Hispalense, Rondense, Seguro-Cazorlense, Nevadense, Almeriense - Al, Ca, Co, Gr, Hu, J, Ma, Se. 
Ophioglossum azoricum C. Presl, Suppl. Tent. Pterid.: 49 (1846) - Comunidades de pastizales húmedos - Isoeto-Nanojuncetea Mesomediterráneo (VR) - Seguro-Cazorlense - J.

Observaciones: Aunque seguimos admitiendo la necesidad de recolectar más material jiennense que confirmen taxativamente la existencia de esta especie en dicha provincia (Salvo, 1982), creemos lógico admitirla de forma provisional a tenor de los juicios de López-González (1982, 1983).

OSMUNDA L., Sp. Pl.: 1063 (1753)

Osmunda regalis L., Sp. Pl. 2: 1065 (1753)

= Aphyllocalpa regalis (L.) Lag., Garc. \& Clem. in Anal. Cienc. 5: 164 (1803)

- Comunidades de sotos en galería (alisedas, fresnedas, etc.) - Característica de Osmundo-Alnion - Termomediterráneo (R), Mesomediterráneo (VR) - Araceno-Pacense, Onubense, Aljíbico - Ca, Hu, Ma. Observaciones: Además de la var. regalis, en Andalucía también se encuentra la var. plumierii (Tausch.) Milde, la cual se caracteriza por tener serrulado el margen de las pínnulas.

$$
\text { POLYPODIUM L., Sp. Pl.: } 1082 \text { (1753) }
$$

Polypodium cambricum L., Sp. P1. 2: 1086 (1753)

= Polypodium australe Fée, Mem. Foug. 5: 236 (1852)

- Comunidades casmofíticas o casmocomofíticas termófilas (raramente epifita) - Característica de Homalothecio-Polypodion (característica de Anogrammo-Polypodietea), Asplenion petrarchae (Asplenietea rupestris) - Termomediterráneo (C), Mesomediterráneo (O), Supramediterráneo (R) - En todas las unidades excepto en Guadiciano-Bacense - Al, Ca, Co, Gr, Hu, J, Ma, Se.

Polypodium canariense Willd., Pr. Tent.: 179 (1836)

= Polypodium macaronesicum Bobrov in Fl. SSSR, Bot. Zurn (Leningrad) 49: 534-535 (1965)

= Polypodium azoricum (Vasc.) Fernandes in Bol. Soc. Brot. 42: 160 (1968)

- Comunidades briopteridofíticas epífitas sobre Quercus canariensis - Frangulo-Rhododendretum baetici - Termomediterráneo (VR) - Aljíbico - Ca.

Observaciones: Tal como se indica en Salvo (1982) la cita de este taxon en nuestra península tan sólo debe referirse al pliego MGC 1125 (cfr. Diez \& Salvo, 1979).

'Polypodium vulgare L., Sp. Pl. 2: 1085 (1753)

- Comunidades de amplias grietas húmedas y umbrías, con un protosuelo tapizado de briófitos - Androsacetalia vandelii (Asplenietea rupestris) - Supramediterráneo (R), Oromediterráneo (R) - Nevadense - Gr.

Polypodium interjectum Shivas in J. Linn. Soc. Bot. 58: 29 (1961)

- Comunidades fisuricolas, esciófilas y humícolas - AnogrammoPolypodietea - Termomediterráneo (VR), Mesomediterráneo (R) - Aljíbico, Bermejense, Seguro-Cazorlense - Ca, J, Ma.

HHIBRIDOS: Polypodium $x$ fontqueri Rothm. in Cadevall \& Font-Quer, Fl. Catalunya 6: 353 (1936) (= P. cambricum x P. vulgare )

Polypodium $x$ shivasiae Rothm. in Rothm. \& Schneider in 
Die Kulturpfl. Deutsch. Akad. Wissen. Berlin 3: 245 (1962) $(=$ P. cambricum $\times$ P. interjectum $)(=$ P. rothmaleri Shivas in Brit. Fern Gaz. 10: 152 (1970).

CHEILANTHES Swartz, Syn. Fil. 5: 126 (1806)

Cheilanthes guanchica Bolle in Bonpladia 7:107 (1859)

- Comunidades que se establecen sobre protosuelos ácidos - Característica de Cheilanthetalia maranto-maderensis (Asplenietea rupestris) - Termomediterráneo (O), Mesomediterráneo (R) - Araceno-Pacense, Marianense, Gaditano, Rondense, Bermejense, Almijaro-Cazulense, Almeriense - Al, Ca, Co, Gr, Hu, J, Ma, Se.

Cheilanthes pteridioides (Reichard) C. Chr., Ind. Fil: 178 (1905)

$\equiv$ Polypodium pteridioides Reichard in L., Syst. Pl.:424 (1780)

= Cheilanthes fragrans (L. fil.) Swartz, Syn. Fil.: 127, 325 (1806)

- Comunidades casmofíticas xerófilas - Característica de Cheilantho pteridioidi-Asplenietum petrarchae (Asplenietalia petrarchae, Asplenietea rupestris) - Termomediterráneo (C), Mesomediterráneo (O) - En todas las unidades excepto en Guadiciano-Bacense - Al, Ca, Co, Gr, Hu, J, Ma, Se.

Cheilanthes maderensis Lowe in Trans. Cambridge Philos. Soc. 6: 528 (1838)

- Comunidades de grietas anchas y pequeños taludes umbríos y húmedos, - Característica de Asplenio-Cheilanthion maderensis y de Cheilanthetalia maranto-maderensis (Asplenietea rupestris) - Termomediterráneo (O), Mesomediterráneo (O) - Araceno-Pacense, Marianense, Rondense, Malacitano-Axarquiense, Alpujarro-Gadorense, Nevadense, Almeriense - Al, Ca, Co, Gr, Hu, J, Ma, Se.

Cheilanthes hispanica Mett. in Abh. Seckenberg. Naturf. Ges. 3: 74 (1859)

- Comunidades rupícolas acidófilas y heliófilas - característica de Buffonio-Cheilanthetum hispanicae y de Cheilanthion hispanicae - Mesomediterráneo (O) - Araceno-Pacense, Andevalense, Marianense, Rondense - Co, Hu, J, Ma, Se.

Cheilanthes tinaei Tod. in Giorn. Sci. Nat. Eco. Palermo 1: 217-218 (1886)

= Cheilanthes $\mathrm{x}$ duriense Mendoça \& Vasconcellos in Anais Inst. Vinho Porto 15 (4): 47 (1956)

= Cheilanthes corsica Reichstein \&. Vida in Candollea 28: 85 (1973)

- Comunidades de grietas amplias, umbrosas en las que se acumula un protosuelo de carácter ácido - Característica de Asplenio billotii-Cheilanthetum tinaei y de Cheilanthenion tinaei (Cheilanthion hispanicae) - Mesomediterráneo ( $R$ ) - Araceno-Pacense, Andevalense, Marianense, Onubense - Co, Hu, J, Se.

HIBRIDOS: Cheilanthes $x$ iberica Rasbach \& Reichstein in Webbia 35: 264 (1982)

Cheilanthes $x$ malacitensis Rasbach \& Reichstein in Webbia 35: 269 (1982) (= C. guanchica $\times$ C. pteridioides) 
NOTHOLAENA R. Brown, Prodr. Fl. Nov. Holl.: 145 (1810)

Notholaena marantae (L.) Desv. in J. Bot. Agric. 1: 92 (1813)

$\equiv$ Acrostichum marantae L., Sp. Pl. 2: 1071 (1753)

= Cheilanthes marantae (L.) Domin in Bibl. Bot. Stuttgart 20: 123, adnot 1 (1915)

- Comunidades de fisuras y protosuelos terrosos de rocas silíceas, serpentínicas o basálticas, fuertemente insoladas - Característica de Cheilanthetum maranto-maderensis - Mesomediterráneo (O) - Bermejense - Ma.

Cosentinia Todaro, Syn. Pl. Vasc. Sic.: 14 (1866)

Cosentinia vellea (Ait.) Todaro, Syn. Pl. Vasc. Sic.: 15 (1866)

$\equiv$ Acrostichum velleum Ait. in Hort. Kew. 3:457 (1789)

= Notholaena vellea (Ait.) Desv. in J. Bot. Agric. 1: 92 (1813)

$=$ Cheilanthes vellea (Ait.) Muller in Frag. Phytogr. Austr. 5: 123 (1866)

$=$ Cheilanthes catanensis (Cosent.) H.P. Fuchs in Brit. Fern Gaz. 9: 9 (1961)

- Comunidades de fisuras o rellanos terrosos de rocas calizas - silicibasícolas, fuertemente insoladas - Asplenietalia glandulosi, caracteristica de Cheilanthetalia maranto-maderensis - Termomediterráneo (C), Mesomediterráneo (O) - Araceno-Pacense, Andevalense, Marianense, Onubense, Hispalense, Rondense, Bermejense, Mijense, Malacitano costero, Malacitano-Axarquiense, Almijaro-Cazulense, Alpujarro-Gadorense, Almeriense - Al, Ca, Co, Gr, Hu, J, Ma, Se.

CRYPTOGRAMMA R. Br. in Frank.,Narr. Journey Polar Sea:767 (1823)

Cryptogramma crispa (L.) R. Br. ex Hooker, Gen. Fil.: tb. 115 b (1842)

$\equiv$ Osmunda crispa L., Sp. Pl. 2: 1067 (1753)

- Comunidades de suelos pedregosos móviles, rocas cascajares y grietas anchas con protosuelo ácido - Holcion cespitosi (Característica de Androsacetalia vandelii) - Oromediterráneo (O) - Nevadense - Gr.

$$
\text { PTERIS L., Sp. Pl.: } 1073 \text { (1753) }
$$

Pteris palustris Poir. in Lam., Encycl. 5: 722 (1804)

= Pteris serrulata auct. plur. non Forsskal

- Comunidades riparias con elevada humedad atmosférica y edáfica, umbrías y con suelo humificado - Frangulo-Rhododendretum baetici (Osmundo-Alnion) - Termomediterráneo (VR) - Aljíbico - Ca.

Pteris vittata L., Sp. Pl. 2: 1074 (1753)

= Pteris ensifolia Poiret in Lam., Encycl. 5: 711 (1804)

- Comunidades de bordes de arroyos, acequias y paredones rezumantes, por los que fluyen aguas básicas - Molinio-Arrhenatheretea, Adiantetea - Termomediterráneo ( $R$ ) - Marianense, Gaditano, Rondense, Bermejense, Mijense, Malacitano-Axarquiense, Malacitano costero, Almijaro-Cazulense, Alpujarro-Gadorense - Al, Ca, Co, Gr, Ma, Se.

$$
\text { ADIANTUM L., Sp. Pl.: } 1094 \text { (1753) }
$$

Adiantum capillus-veneris L., Sp. Pl.: 1096 (1753)

- Comunidades de fisuras calizas rezumantes - Caracteristica 
Adiantetea - Termomediterráneo (C), Mesomediterráneo (C), Supramediterráneo (O) - En todas las unidades - $\mathrm{Al}, \mathrm{Ca}, \mathrm{Co}, \mathrm{Gr}$, Hu, J, Ma, Se.

ANOGRAMMA Link, Fil. Sp. Cultae: 137 (1841)

Anogramma leptophylla (L.) Link, Fil. Sp. Cultae: 137 (1841)

$\equiv$ Polypodium leptophyllum L., Sp. Pl. 2: 1092 (1753)

= Gymnogramma leptophylla (L.) Swartz, Syn. Fil. 23: 218 (1806)

- Comunidades briopteridofíticas algo esciófilas y de ambiente mesofítico proporcionado por los taludes de los bosques templados, de carácter edáfico indiferente - Característica de Anogrammo-Selaginelletum (caracteristica de Anogrammo-Polypodietea) - Termomediterráneo (O), Mesomediterráneo (O), Supramediterráneo (O) - En todas las unidades excepto en la Guadiciano-Bacense - Al, Ca, Co, Gr, $\mathrm{Hu}, \mathrm{J}, \mathrm{Ma}$, Se.

$$
\text { MARSILEA L., Sp. Pl.: } 1099 \text { (1753) }
$$

Marsilea strigosa Willd., Sp. Pl. 5:540 (1810)

- Comunidades herbáceas de desarrollo anual, propias de lagunazos y bordes de lagunas temporales, sobre suelos areno-humosos, silíceos y gleizados - Característica de Isoetalia (Isoeto-Nanojuncetea) - Termomediterráneo (VR) - Marianense (?), Almeriense - Al, Co (?).

VANDENBOSCHIA Copeland in Philip. Journ. Sci. 67: 51 (1938)

Vandenboschia speciosa (Willd.) Kunkel in Ber. Schweiz. Bot. Ges. 76: 48 (1966)

$\equiv$ Trichomanes speciosa Willd., Sp. Pl. 5: 514 (1810)

- Comunidades de taludes terrosos próximos a escorrentías de aguas, de tal forma que asegura mediante la sálpicadura una elevada humedad ambiental - Frangulo-Rhododendretum baetici (Osmundo-Alnion) - Termomediterráneo (VR) - Aljíbico - Ca.

Culcita C. Presl, Tent. Pterid.: 135 (1836)

Culcita macrocarpa C. Presl, Tent. Pterid.: 135 (1836)

- Comunidades de sotobosques ribereños umbrios, húmedos y con escasas variaciones térmicas - Característica de Frangulo-Rhododendretum baetici (Osmundo-Alnion) - Termomediterráneo (VR) Aljíbico - Ca.

PTERIDIUM Gledtisch ex Scopoli, Fl. Carniol.: 169 (1760)

Pteridium aquilinum (L.) Kuhn in Decken, Reisen Ost. Africa 3(3): 11 (1879)

$\equiv$ Pteris aquilina L., Sp. Pl.: 1075 (1753)

= Pteris heredia Clem. ex Colm., Enum. Cript. Esp. y Port. 1: 16 (1867)

- En sotobosques, sobre suelos profundos, generalmente ácidos, gleizados - Oleo-Quercion rotundifoliae suberis, Quercion fagineo-suberis - Termomediterráneo (C), Mesomediterráneo (C), Supramediterráneo (O) - En todas las unidades corológicas excepto Almeriense y Guadiciano-Bacense - Ca, Co, Gr, Hu, J, Ma, Se.

Observaciones: Siguiendo a Salvo (1982) reconocemos dos subespecies para Andalucia: Subsp. aquilinum y subsp. brevipes (Tausch) Vul'f, Fl. Krymea 1(1): 20 (1927). La aceptación de este segundo 
taxon se basa en la cita de un citótipo tetraploide para la Sierra de Cazorla (Löve \& Kjellqvist, 1972).

THELYPTERIS Schmidel, Icon. Pl. ed. Keller: 45 (1763)

Thelypteris palustris Schott, Gen. Fil.: t. 10 (1834)

= Thelypteris thelypteroides (Michx.) Holub in Taxon 21: 332 (1972) var. glabra Holub in Taxon 21: 332 (1972)

- Comunidades de márgenes de ríos y de remansos de agua dulce, sobre suelos higroturbosos (cañaverales, juncales o incluso alisedas) - Caracteristica de Caricetum pseudocypero-lusitanicae (Phragmitetalia), Osmundo-Alnion (Querco-Fagetea), HoloschoenoJuncetum acuti (Molinio-Arrhenatheretea) - Termomediterráneo (VR) Onubense, Aljíbico - Ca, Hu.

CHRISTELLA Léveillé, Fl. Kouy-Tchéou: 472 (1915)

Christella dentata (Forsskal) Brownsey \& Jermy in Brit. Fern Gaz 10: 338 (1973)

ミPolypodium dentatum Forsskal, Fl. Aegypt.-Arab.: 185 (1775)

$=$ Cyclosorus dentatus (Forsskal) R. Ching in Bull. Fam. Mem.

Inst. Brol. (Peking) Bot. 8: 206 (1938)

-En comunidades de suelos fangosos, protegidos por una densa cobertura arbórea - Zona ecotónica entre Osmundo-Alnion y MyrtoQuercetum suberis - Termomediterráneo (VR) - Aljíbico - Ca.

$$
\text { ASPLENIUM L., Sp. Pl.: } 1078 \text { (1753) }
$$

Asplenium subglandulosum (Hooker \& Greville) Salvo, Prada \& Diaz in Candollea 37: 469 (1982) subsp. hispanicum (Cosson) Salvo, Prada \& Diaz in Candollea 37:481 (1982)

E Grammitis hispanica Cosson, Not. Pl. Crit.: 48 (1849)

= Pleurosorus hispanicus (Cosson) Morton in Bull. Soc. Bot. France 106: 233 (1959)

= Pleurosorus nevadensis Salvo in Lazaroa 1: 147 (1980)

- Comunidades casmocomofíticas de fisuras y grietas profundas de rocas básicas - Asplenion glandulosi (Asplenietea rupestris) Mesomediterráneo (O), Supramediterráneo (R) - Rondense, AlmijaroCazulense, Granatense, Nevadense - Ca, Co, Gr, J, Ma, Se.

Asplenium ceterach L., Sp. P1. 2: 1080 (1753)

= Ceterach officinarum DC. in Lam. \& DC., Fl. Franç. (éd. 3) 2: 566 (1805)

- Comunidades casmofíticas, heliófilas, subnitrófilas, frecuentemente sobre sustratos básicos - Característica de Parietarietea judaicae, característica de Asplenietea rupestris - Termomediterráneo (VC), Mesomediterráneo (C), Supramediterráneo (O), Oromediterráneo (O) - En todas las unidades corológicas excepto en la Onubense - Al, Ca, Co, Gr, Hu, J, Ma, Se.

Asplenium marinum L., Sp. Pl. 2: 1081 (1753)

- Comunidades de fisuras de acantilados marinos - Característica de Asplenietum marini (Crithmo-Limonietea) - Termomediterráneo (VR) - Gaditano - Ca.

Asplenium viride Hudson, Fl. Angl.: 385 (1762)

- Comunidades de grietas protegidas o de escasa insolación silicícola - Androsacetalia vandelii - Oromediterráneo (O) - Nevadense - Gr. 
Asplenium trichomanes L., Sp. Pl. 2: 1080 (1753), subsp. quadrivalens Meyer in Ber. Deutschl. Bot. Ges. 74: 456 (1962)

- Comunidades casmocomofíticas - Característica de Asplenietea rupestris, Característica de Parietarietea judaicae - Termomediterráneo (VC), Mesomediterráneo (C), Supramediterráneo (C) - En todas las unidades excepto en Onubense y Guadiciano-Bacense - Al, $\mathrm{Ca}, \mathrm{Co}, \mathrm{Gr}$, Hu, J, Ma, Se.

Asplenium trichomanes L., Sp. P1. 2: 1080 (1753) subsp. pachyrrachis (Christ) Lovis \& Reichstein in Wildenowia 10: 18 (1980)

$\equiv$ Asplenium trichomanes L. sublusus pachyrrachis Christ in Beitr. Kryptogrammenfl. Schweiz. 1(2): 92 (1900)

= Asplenium csikii Kürmmeler \& Andrasovszky in Magiar Bot. Lapok 21: 3 (1923)

- Comunidades fisurícolas calcícolas de alta montaña - Asplenion petrarchae (Asplenietea rupestris) - Mesomediterráneo (VR), Supramediterráneo (R) - Nevadense, Rondense, Seguro-Cazorlense Gr, J, Ma.

Asplenium petrarchae (Guerin) DC. in Lam. \& DC., Fl. Franç. (3 ed.) 5: 238 (1815) subsp. petrarchae

$\equiv$ Polypodium petrarchae Guerin, Descr. Fontain Vaucluse 1: 124 (1804)

= Asplenium glandulosum Loisel., Notice: 145 (1810)

- Comunidades fisuricolas heliófilas y basófilas - Caracteristica de Asplenion petrarchae (Asplenietalia petrarchae, Asplenietea rupestris) - Termomediterráneo (O), Mesomediterráneo (C), Supramediterráneo (R) - Araceno-Pacense, Gaditano, Rondense, Malacitanocostero, Malacitano-Axarquiense, Almijaro-Cazulense, Alpujarro-Gadorense, Nevadense, Granatense, Filabrido-Bacense, Marianense, Seguro-Cazorlense - Al, Ca, Co, Gr, Hu, J, Ma, Se.

Asplenium petrarchae (Guerin) DC. in Lam. \& DC., Fl. Franç. (3 ed.) 5: 238 (1815) subsp. bivalens (Meyer) Lovis \& Reichstein in Ber. Schweiz. Bot. Ges. 79: 336 (1969)

$\equiv$ Asplenium glandulosum Loisel. subsp. bivalens Meyer in Ber. Deutschl. Bot. Ges. 77: 7 (1964)

= Asplenium glandulosoides Löve \& Löve in Taxon 26: 326 (1974)

- Comunidades fisuricolas heliófilas y basófilas - Asplenion petrarchae (Asplenietea rupestris) - Mesomediterráneo (VR) - Rondense - Ca, Se.

Asplenium adiantum-nigrum L., Sp. Pl. 2: 1081 (1753)

- Comunidades de fisuras de rocas y de taludes terrosos, umbrófilas - Asplenietea rupestris - Mesomediterráneo (R), Supramediterráneo (R), Oromediterráneo (R) - Nevadense, Bermejense - Gr, Ma.

Observaciones: En Andalucía además de la variedad típica, también se encuentra la variedad corunnense Christ in Merino, Fl. Galic. 3: 494 (1909). A esta variedad, que coloniza sustratos ultrabásicos, hay que referir cuantas citas ibéricas se han realizado de $\mathbf{A}$. cuneifolium Viv.; así como las de A. x centovallense Meyer dadas para el sector bermejense (Salvo,1982).

Asplenium onopteris L., Sp. Pl. 2: 1081 (1753)

- Comunidades esciófilas, sobre suelos profundos, preferentemente ácidos - Característica de Quercetalia ilicis (Quercetea ilicis), Anogrammo-Polypodietea, Phagnalo-Rumicetea indurati - Termomediterráneo (O), Mesomediterráneo (C) - Araceno-Pacense, Marianense, Gaditano, Aljíbico, Rondense, Almijaro-Cazulense, Alpujarro-Gadoren- 
se, Seguro-Cazorlense, Nevadense - Al, Ca, Co, Gr, Hu, J, Ma, Se.

Asplenium fontanum (L.) Bernh. in J. Bot. Schrader 1: 314 (1799)

$\equiv$ Polypodium fontanum L., Sp. Pl. 2: 1089 (1753)

- Comunidades casmofíticas, calcícolas, de tendencia esciófila - Potentilletalia caulescentis - Mesomediterráneo (VR) - Seguro-Cazorlense - J.

Asplenium foreziense Heribaud in Magnier, Fl. Select. Exsicc. no. 743 (In schedis distr. 1884 ex Magnier: Scrinia Florae Selectae 3: 65. 1884)

- Comunidades pioneras de fisuras húmedas de rocas silíceas - Androsacetalia vandelii - Supramediterráneo (R) - Nevadense, Filabrido-Bacense - Al, Gr.

Asplenium billotii F. Schultz in Flora 28: 738 (1845)

- Comunidades de fisuras de rocas y taludes terrosos, umbrías y generalmente silicícolas - Característica de Asplenio-Cheilanthion maderensis (Asplenietea rupestris) - Termomediterráneo (O), Mesomediterráneo (C) - Araceno-Pacense, Andevalense, Marianense, Onubense, Gaditano, Aljíbico, Rondense, Bermejense, Almeriense - Al, Ca, Co, Hu, J, Ma, Se.

Asplenium ruta-muraria L., Sp. Pl. 2: 1081 (1753)

- Comunidades pioneras de paredones verticales rocosos, grietas y fisuras de rocas - Caracteristica de Asplenietea rupestris Mesomediterráneo (R), Supramediterráneo (O), Oromediterráneo (O) - Almijaro-Cazulense, Rondense, Alpujarro-Gadorense, Nevadense, Filabrido-Bacense, Seguro-Cazorlense, Marianense - Al, Gr, J, Ma, Se.

Asplenium seelosii Leybold in Flora 38: 348 (1855) subsp. glabrum (Litard. \& Maire) Rothm. in Cadevall, F1. Catalunya 6: 339 (1937)

Asplenium seelosii Leybold var. glabrum Litard. \& Maire in Maire, Cont. Etude Fl. Afrique Nord 13: 143 (1929)

= Asplenium celtibericum Rivas-Martínez in Bull. Jard. Bot. Natl. Belgique 37: 329 (1967)

- Comunidades fisurícolas de rocas dolomíticas extraplomadas - Asplenietea rupestris - Oromediterráneo (VR) - Seguro-Cazorlense - J.

Asplenium septentrionale (L.) Hoffm. in Deutschl. Fl. 2: 12 (1796)

$\equiv$ Acrostichum septentrionale L., Sp. Pl. 2: 1068 (1753)

- Comunidades de fisuras de rocas verticales y poco expuestas - Androsacetalia vandelii (Asplenietea rupestris) - Oromediterráneo (O) - Nevadense - Al, Gr.

Asplenium scolopendrium L., Sp. Pl.: 1079 (1753)

= Phyllitis scolopendriuril (L.) Newman, Hist. Brit. Fern (2 ed.): 10 (1844)

- Comunidades de grietas anchas en rocas umbrosas, muros rezumantes, bosques sombríos y frescos - Adiantetea - Mesomediterráneo (R), Supramediterráneo (R) - Marianense, Rondense, Nevadense, Seguro-Cazorlense, Almijaro-Cazulense - Ca, Gr, J, Ma, Se.

Asplenium sagittatum (DC.) A. J. Bange in Bull. Mem. Soc. Linn. Bot. Lyon 21: 84 (1952)

$=$ Phyllitis sagittata (DC.) Guinea \& Heywood in Collectanea Botanica 4: $246(1954)$ 
- En grietas profundas de rocas calizas - Adiantetea - Termomediterráneo (R), Mesomediterráneo (VR) - Gaditano, Rondense - Ca.

HIBRIDOS: Asplenium $x$ nieschalkii Meyer in Ber. Deutsch. Bot. Ges. 76: 18 (1963) (= A. trichomanes subsp. quadrivalens $x$ A. petrarchae subsp. petrarchae).

Asplenium $x$ costei Litard. in Notes pteridologiques: Bull. Acad. Inst. Geogr. Bot. (Le Mans) 21: 150. (1911) (= A. foreziense $x$ A. septentrionale).

CYSTOPTERIS Bernh. in Neues J. Bot. 1 (2): 26 (1805)

Cystopteris fragilis (L.) Bernh. in Neues J. Bot. 1: 27 (1806)

$\equiv$ Polypodium fragile L., Sp. Pl. ( 2 ed.): 1553 (1763)

- Comunidades de fisuras de rocas y muros frescos - Anogrammo-Polypodietea, Asplenietea rupestris, Parietarietea judaicae Mesomediterráneo (O), Supramediterráneo (O), Oromediterráneo (C) - Araceno-Pacense, Marianense, Rondense, Almijaro-Cazulense, Alpujarro-Gadorense, Granatense, Filabrido-Bacense, Nevadense, SeguroCazorlense - Al, Ca, Co, Gr, Hu, J, Ma.

Observaciones: En Andalucía existen dos variedades, la variedad fragilis, que es la más frecuente y la variedad huteri (Haussmann) Milde, caracterizada por el margen foliar glanduloso y que aparece restringida a las altas cumbres calizas nevadense.

Cystopteris dickieana R. Sim. in Gard. Farmer's J. 2 (20): 308 (1848)

- Comunidades de fisuras de rocas calizas - Anogrammo-Polypodietea, Asplenietea rupestris - Mesomediterráneo (O), Supramediterráneo (R) - Rondense, Granatense, Nevadense, Filabrido-Bacense, Seguro-Cazorlense - Al, Ca, Gr, J.

Cystopteris viridula (Desv.) Desv. in Mem Soc. Linn. Paris 6: 264 (1827)

$\equiv$ Aspidium viridulum Desv. in Ges. Natur. Srunde Berlin Mag. 5: 321 (1811)

- Comunidades de taludes terrosos húmedos próximos a cauces de aguas - Osmundo-Alnion - Mesomediterráneo (VR) - Araceno-Pacense - Hu.

ATHYRIUM Roth, Tent. Fl. Germ. 3: 31 (1799)

Athyrium filix-femina (L.) Roth, Tent. Fl. Germ. 3: 65 (1799)

$\equiv$ Polypodium filix-femina L., Sp. Pl. 2: 1090 (1753)

- Comunidades umbrías de bordes de arroyos - Osmundo-Alnion, Populion albae - Termomediterráneo (C), Mesomediterráneo (O), Supramediterráneo (O) - Araceno-Pacense, Aljíbico, Bermejense, Nevadense - Al, Ca, Gr, Hu, Ma.

DIPLAZIUM Swartz in J. Bot. Schrader 1800 (2): 61 (1801)

Diplazium caudatum (Cav.) Jermy in Brit. Fern Gaz. 9: 161 (1964)

झ Tectaria caudata Cav. in Anales Cienc. Nat. 4:100. 1801.

- Comunidades de barrancos profundos, muy umbrios y húmedos - Frangulo-Rhododendretum baetici (Osmundo-Alnion) - Termomediterráneo (VR) - Aljíbico - Ca. 
DRYOPTERIS Adanson, Fam. Pl. 2: 20, 551 (1763)

Dryopteris filix-mas (L.) Schott, Gen. Fil.: tab. 9 (1834)

$\equiv$ Polypodium filix-mas L., Sp. Pl. 2: 1090 (1753)

- Comunidades rupícolas, glerícolas, boscosas, etc. - Populion albae, Osmunso-Alnion, Androsacetalia vandelii - Termomediterráneo (R), Oromediterráneo (O) - Marianense, Seguro-Cazorlense, Aljíbico, Nevadense - Al, Ca, Co, Gr, J.

Dryopteris affinis (Lowe) Fraser-Jenkins in Fern Gaz. 12: 56 (1979) subsp. affinis

$\equiv$ Nephrodium affine Lowe in Trans. Cambridge Philos. Soc. 6: 525 (1838)

- Comunidades boscosas, umbrías y húmedas, sobre suelos ácidos - Osmundo-Alnion - (Mesomediterráneo (VR) - Araceno-Pacense, Aljíbico (?), Nevadense (?) - Ca (?), Gr (?), Hu.

Dryopteris affinis (Lowe) Fraser-Jenkins in Fern Gaz. 12: 56 (1979)

subsp. borreri (Newman) Fraser-Jenkins in Willdenowia 10: 110 (1980)

$\equiv$ Dryopteris filix-mas var. borreri Newman, Hist. Brit. Ferns 3: 189 (1854)

= Dryopteris borreri (Newman) Oberholzer \& Tavel in Verh. Schweiz. Naturf. Ges. 118: 153 (1937)

= Dryopteris pseudomas (Wollaston) Holub \& Pouzar in Folia Geobot. Phytotax. Bohem. 2: 232 (1967)

- Comunidades boscosas, umbrías y húmedas, sobre suelos ácidos - Populion albae - Supramediterráneo (R) - Nevadense - Gr.

Dryopteris submontana (Fraser-Jenkins \& Jermy) Fraser-Jenkins in Candollea 32: 311 (1977)

$\equiv$ Dryopteris villari (Bellardi) Waynar ex Schinz \& Tell subsp. submontana Fraser-Jenkins \& Jermy in Fern Gaz. 11: 838 (1977)

- Comunidades de grietas y gleras de rocas calizas - Caracteristica de Dryopteridion submontanae - Supramediterráneo ( $R$ ) Almijaro-Cazulense, Nevadense, Seguro-Cazorlense - Gr, J, Ma.

Dryopteris tyrrhena Fraser-Jenkins \& Reichstein in Fern Gaz. 11: 80 (1975)

= Aspidium nevadense Boiss., Elench. Pl. Nov.: 93 (1838)

- Comunidades de gleras esquistosas próximas a bordes de arroyos - Androsacetalia vandelii - Supramediterráneo (VR) - Nevadense - Gr.

Dryopteris guanchica Gibby \& Jermy in Bot. J. Linn. Soc. 74: 256 (1977)

Citado del sector aljíbico por Fraser-Jenkins (1982), basándose para ello en un pliego recolectado por Ball en 1851, sin que haya sido confirmado posteriormente. Ca (?).

POLYSTICHUM Roth, Tent. F1. Germ. 3: 31 (1799)

Polystichum lonchitis (L.) Roth, Tent. Fl. Germ. 3: 71 (1799)

$\equiv$ Polypodium lonchitis L., Sp. Pl.: 1088 (1753)

- Comunidades glerícolas y fisuricolas - Thlaspetea rotundifo-

liae (?) - Oromediterráneo (C) - Nevadense, Seguro-Cazorlense $\mathrm{Al}, \mathrm{Gr}, \mathrm{J}$. 
Polystichum setiferum (Forsskal) Woynar in Mitt. Naturwiss. Vereines Steiermark 49: 181 (1913)

$\equiv$ Polypodium setiferum Forsskal, Fl. Aegypt.- Arab.: 185 (1775)

- Comunidades de bosques ribereños umbrosos y permanentemente húmedos - Característico de Osmundo-Alnion - Termomediterráneo (O), Mesomediterráneo (R) - Araceno-Pacense, Aljíbico - Ca, $\mathrm{Hu}, \mathrm{Ma}$.

Polystichum aculeatum (L.) Roth in Arch. Bot. (Leipzig) 2: 106 (1799)

$\equiv$ Polypodium aculeatum L., Sp. Pl.: 1090 (1753)

- Comunidades umbrosas y frescas próximas a corrientes de aguas - Populetalia albae, Thlaspetea rotundifoliae (?) - Mesomediterráneo (O), Supramediterráneo (O), Oromediterráneo (O) - Rondense, Almijaro-Cazulense, Nevadense, Seguro-Cazorlense - Al, Gr, J, Ma.

HIBRIDOS: Polystichum $x$ illyricum (Borbás) Hahne in Allg. Bot. Z. Syst. 10: 103 (1904) (= P. lonchitis X P. aculeatum).

DAVAllia Sm. in Mem. Acad. Roy. Sci (Turin) 5: 414 (1793)

Davallia canariensis (L.) Sm. in Mém. Acad. Sci. (Turin) 5: 414 (1793)

$\equiv$ Trichomanes canariense L., Sp. Pl.: 1099 (1753)

= Polypodium lusitanicum L., Sp. Pl.: 1094 (1753)

- Comunidades briopteridofíticas epifíticas o epilíticas - Rusco-Quercetum canariensis, Frangulo-Rhododendretum baetici - Termomediterráneo (O) - Aljíbico, Bermejense - Ca, Ma.

$$
\text { BLECHNUM L., Sp. P1.: } 1077 \text { (1753) }
$$

Blechnum spicant (L.) Roth in Ann. Bot. (Usteri) 10: 56 (1794)

$\equiv$ Osmunda spicant L., Sp. Pl. 2: 1066 (1753)

- Comunidades de bosques ribereños, umbríos y frescos - Osmundo-Alnion - Termomediterráneo (R), Mesomediterráneo (R) - Araceno-Pacense, Aljíbico - Ca, Hu.

$$
\text { AZOLla Lam., Encycl. 1: } 343 \text { (1783) }
$$

Azolla filiculoides Lam., Encycl. 1: 343 (1783)

- Charcas de aguas poco profundas - Lemnetea - Mesomediterráneo (VR) - Marianense - Co.

\section{BIBLIOGRAFIA}

DIEZ, B. \& SALVO, A.E. -1979- Sobre la existencia de Polypodium macaronesicum en el S de la Península Ibérica. Acta Bot. Malacitana 5: 5-14.

FRASER-JENKINS, C. -1982- Dryopteris in Spain, Portugal ánd Macaronesia. Bol. Soc. Brot. 55: 175-336.

LOPEZ-GONZALEZ, G. -1982- La distribución en España de Ophioglossum azoricum Presl. Anales Jard. Bot. Madrid 38: 524-525.

LOPEZ-GONZALEZ, G. -1983- Ophioglossum azoricum C. Presl en Navodres, provincia de Cuenca (España). Anales Jard. Bot. Madrid 40: 280281.

LOVE, A. \& KJELLQVIST, E. -1972- Cytotaxonomy of Spanish plants. I. In- 
A. E. SALVO \& B. CABEZUDO

troduction. Pteridophyta and Gymnospermae. Lagascalia 2: 23-35.

MOLESWORTH, B. -1968- Observations on two spanish ferns. Brit. Fern Gaz. 10: $34-36$.

PRADA, C. -1983- El género Isoetes L. en la Península Ibérica. Acta Bot. Malacitana 8:73-100.

RIVAS-MARTINEZ, S., COSTA, M., IZCO, J. \& SAENZ, C. -1981- Flora Matritensis, I (Pteridophyta). Lazaroa 3:25-61.

SALVO, A.E. -1982- Flora pteridofítica de Andalucía. Pub. Dep. Bot. Univ. Málaga.

WILLMANNS, 0 \& H. RASBACH -1973- Observations on the pteridophyta of São Miguel, Açores. Brit. Fern Gaz. 10: 315-329. 